\title{
SUBESCALA OCS-CBCL DE NELSON PARA LA EVALUACIÓN DEL TRASTORNO OBSESIVO-COMPULSIVO INFANTO-JUVENIL: ANÁLISIS DE VALIDEZ EN UNA MUESTRA ESPAÑOLA
}

\author{
Santiago Batlle, Lurdes Duñó, Ester CAmprodon, Xavier Estrada, \\ MARTA ACEÑA, Elena Pujals, y Luis Miguel Martin
}

Centro de Salud Mental Infantil y Juvenil Sant Martí Sud, Barcelona, España Universidad Autónoma de Barcelona, España

\begin{abstract}
Resumen: El objetivo del estudio es investigar la utilidad de la escala obsesivo-compulsiva basada en el Child Behavior Checklist, OCS-CBCL (Nelson et al., 2001). Se determina la consistencia interna, la sensibilidad, la especificidad y el valor predictivo positivo y negativo para identificar el trastorno obsesivo-compulsivo (TOC) en tres muestras de niños y adolescentes $(N=26)$ apareadas por edad y género: (1) pacientes con TOC, (2) pacientes psiquiátricos sin TOC, (3) población general. El ANOVA muestra diferencias significativas entre grupos $(p<0,001)$ y el análisis pot-hoc de Scheffé diferencias significativas del grupo TOC con los otros dos grupos. La sensibilidad es del $73,1 \%$ al $76,9 \%$, la especificidad del $78,8 \%$ al $86,5 \%$, el VPP del $78,4 \%$ al $84,4 \%$ y el VPN del $76,3 \%$ al 77,3\%. Los resultados sugieren la utilidad de la OCS-CBCL para la detección del TOC infanto-juvenil.
\end{abstract}

Palabras clave: Adolescencia; CBCL; evaluación; infancia; ROC; trastorno obsesivo-compulsivo.

\section{Nelson's OCS-CBCL Subscale for the assessment of Obsessive-Compulsive Disorder (OCD) in children and adolescents: Validity analysis in a Spanish sample}

\begin{abstract}
The aim of this study was to investigate the usefulness of the obsessive-compulsive scale based upon the Child Behavior Checklist, OCS-CBCL (Nelson et al., 2001). We determined internal consistency, sensitivity, specificity and positive and negative predictive value to identify obsessive-compulsive disorder (OCD) in three samples of children and adolescents $(\mathrm{N}=26)$ matched for age and gender: (1) patients with OCD, (2) psychiatric patients without OCD, and (3) general population. The ANOVA revealed significant differences between groups $(p<0,001)$ and post-hoc Scheffé analysis showed significant differences between the OCD group and the other two. The sensitivity was $73.1 \%$ to $76.9 \%$, specificity $78.8 \%$ to $86.5 \%$, PPV $78.4 \%$ to $84.4 \%$ and NPV was $76.3 \%$ to $77,3 \%$. The results suggest the usefulness of the OCS-CBCL for the detection of child and adolescent OCD.
\end{abstract}

Keywords: Adolescence; assessment; CBCL; childhood; obsessive-compulsive disorder.

\section{INTRODUCCIÓN}

El trastorno obsesivo-compulsivo (TOC) es un trastorno de ansiedad que perturba significativamente el funcionamiento académico, so-

\footnotetext{
Recibido: 27 septiembre 2011; aceptado: 11 diciembre 2011.

Correspondencia: Santiago Batlle Vila, RamonTurró 337, 3. planta, 08019 Barcelona. Correo-e: sbatlle.bcn.ics@, gencat.cat
}

cial y profesional del sujeto. La principal característica de este trastorno es la presencia recurrente de obsesiones y compulsiones que interfieren en la vida cotidiana de la persona.

Los datos obtenidos por diferentes estudios epidemiológicos aportan información referente a la posibilidad que, en este trastorno, exista una tasa de prevalencia real mayor de la observada clínicamente; poniendo de manifiesto que al menos un $80 \%$ de los pacientes adultos diagnosticados de TOC iniciaron el trastorno duran- 
te su infancia, y que el primer pico de máxima incidencia ocurre entre los 12 y los 14 años de edad. Así mismo, estudios en adolescentes han observado tasas de prevalencia del TOC que van del $1 \%$ al $4 \%$, con registros de tasas de comorbilidad de hasta en un $70 \%$ de los pacientes y un curso crónico en más del $40 \%$ de los jóvenes (Flament et al., 1998; Helbing y Ficca, 2009; Walitza et al., 2011).

Entre los adolescentes con TOC, la literatura indica que muy pocos reciben un diagnóstico correcto, y menos aún reciben un tratamiento adecuado (Helbing y Ficca, 2009) en un momento en el que los modelos metodológicos y de tratamiento que se pretenden validar y aplicar en los servicios públicos de salud mental deberían estar basados en resultados empíricos y evidencia científica (Moriana y Martínez, 2011). Estos datos indican la necesidad de afinar en el diagnóstico; pues, si bien disponemos de tratamientos cognitivos, conductuales (Turner, 2006) y farmacológicos eficaces; estudios de seguimiento a nueve años señalan que entre el 40-56\% de jóvenes siguen teniendo síntomas clínicamente significativos (el 31\% síntomas relevantes, un $13 \%$ síntomas moderados y un $11 \%$ síntomas graves),y que el $44 \%$ presentan síntomas subclínicos (Black y Gaffney, 2008; Bloch et al., 2009; Micali et al., 2010).

La identificación de fenomenología obsesivo-compulsiva en síndromes autoinmunes y enfermedades neurológicas, ha despertado el interés, en pediatras y neuropediatras, ante la necesidad de realizar un diagnóstico diferencial del trastorno obsesivo-compulsivo (Bernstein, Victor, Pipal y Williams, 2010; Lin et al., 2010; Schrag et al., 2009).

Una de las mayores dificultades ante las que se enfrenta el clínico en la evaluación de síntomas del TOC, es poder diferenciar los rituales propios de la edad (rutinas y preocupaciones ansiosas) de los rituales patológicos (Belloch, Cabedo, Morillo, Lucero y Carrió, 2003) y los pensamientos obsesivos de las rumiaciones presentes en otros trastornos de ansiedad (GarcíaSoriano, Belloch, y Morillo, 2008; Sandín, Chorot, Valiente y Lostao; 2009). Esta dificultad es mayor en la infancia (Carter, Pauls y Leckman, 1995; Evans, Leckman, Carter y Reznick, 1997; King y Noshpitz, 1991; Leonard, 1989;
Leonard, Goldberger, Rapoport., Cheslow y Swedo, 1990) debido a que, a menudo, los niños presentan preocupaciones extremas ante circunstancias cotidianas cuya frecuencia e intensidad no son patológicas. Otra dificultad diagnóstica reside en el hecho que frecuentemente los niños se muestran reservados acerca de los síntomas obsesivo-compulsivos, siendo frecuente que intenten minimizarlos o negarlos. Además, el 75\% de los pacientes con TOC presentan otros síntomas comórbidos que pueden enmascarar el trastorno principal (Geller et al., 2000; Hanna, 1995; Ivarsson, Melin y Wallin, 2008).

Otra de las causas que contribuyen en la difícil tarea de diagnosticar el TOC infantil es la limitación en la disponibilidad de instrumentos de detección propios (Batlle et al., 2005). En la actualidad los instrumentos más comúnmente utilizados para evaluar y cuantificar los síntomas TOC son instrumentos validados en adultos que han sido revisados y adaptados para su uso en infancia y adolescencia, como por ejemplo la Children's Yale-Brown Obsessive Compulsive Scale (CY-BOCS), escala aplicada mediante evaluador (Scahill et al., 1997), y el Leyton Obsessional Inventory-Child Version (LOI-CV), cuestionario auto y heteroaplicado (Storch et al., 2010).

La CY-BOCS ha demostrado una buena fiabilidad y validez para la identificación de la gravedad de los síntomas del TOC, pero no es un instrumento de cribado (Storch et al., 2006a). Así mismo, estudios relativos al LOICV muestran una sensibilidad y especificidad aceptables (75\%-88\% y 77\%-84\%, respectivamente, dependiendo del punto de corte utilizado), pero un modesto valor predictivo positivo (VPP) de entre un 15\%-18\% (Bamber, Tamplin, Park, Kyte y Goodyer, 1988; Berg, Whitaker, Davies, Flament y Rapoport, 2002). Un reciente estudio de validación de la LOI-CV en castellano obtuvo, con un punto de corte de 12 , una sensibilidad de $69,5 \%$, una especificidad del 77,7\%, un valor predictivo positivo (VPP) del $16 \%$ y un valor predictivo negativo (VPN) del 96,5\% (Rueda et al., 2007). Para que un instrumento sea considerado un buen instrumento de cribado debe tener un VPP aceptable y un alto VPN. 
El Child Behavior Checklist (CBCL) de Achenbach (1991) es un cuestionario de sencilla aplicación y fácil comprensión para los padres, que se ha convertido en uno de los instrumentos de screening más utilizados en psiquiatría y psicología clínica, tanto en la evaluación de niños como de adolescentes. Es un instrumento fácil y comprensible para los padres y de sencilla corrección para los clínicos, pues dispone de un programa informatizado que permite generar puntuaciones para las diferentes escalas. Estas escalas están estandarizadas según edad y sexo. Además, la fiabilidad, validez y estabilidad temporal de las escalas del CBCL está bien documentada. Existen numerosas publicaciones que han estudiado las propiedades psicométricas de la escala y que concluyen que el CBCL es un instrumento útil para evaluar el comportamiento de un niño, a través de la información de los padres, en un entorno clínico o de investigación. (Achenbach, 1991, Sardinero, Pedreira, y Muñiz, 1997).

En los últimos años, las investigaciones se dirigen hacia la utilización del CBCL como herramienta de detección de los trastornos incluidos en Manual Diagnóstico y Estadístico de los Trastornos Mentales (DSM), (Achenbach y Dumenci, 2001; Achenbach y Rescorla, 2001; Achenbach y Rescorla, 2007). Siguiendo esta línea, un grupo de investigadores del departamento de psiquiatría de la Universidad de Washington desarrollaron una subescala del CBCL para el cribado del TOC en la infancia y la adolescencia (OCS-CBCL) a partir del análisis factorial de los items del CBCL con niños y adolescentes (Nelson, Hanna, Hudziak, Botteron, Heath y Todd, 2001). Los autores iniciaron su trabajo con la identificación de 11 items del CBCL que fueron considerados como los más significativos para el diagnóstico de TOC; conservando, después de un segundo análisis factorial, 8 items (véase Tabla 1) que sometieron a un análisis ROC (Receiver Operating Characteristic).

El análisis de curvas ROC se ha consolidado como el método más adecuado para contrastar la capacidad diagnóstica de un test con un resultado cuantitativo. De entre sus mayores bondades cabe destacar que la medida de precisión diagnóstica que proporciona es independiente de la prevalencia y del punto de corte, permitiendo la comparación directa de los resultados de diferentes estudios realizados sobre una misma prueba diagnóstica. Consecuentemente el área bajo la curva, en inglés area under the curve (AUC), se ha convertido en el índice de resumen de una curva ROC.

La OCS-CBCL ha demostrado una adecuada consistencia interna (alfa de Cronbach $=$ 0,84 ), altos niveles de sensibilidad (del 75,3\% al 84,9\%), una buena especificidad (del 82,2\% al $92,5 \%$ ), un VPP de entre $70,5 \%$ y $83,3 \%$, y VPN de entre $88,2 \%$ y $91,6 \%$; obteniendo además por medio del análisis ROC una AUC =0,96 (Nelson et al., 2001). En los últimos años se han realizado diferentes estudios con esta escala (Althoff, Rettew, Boomsma y Hudziak, 2009; Geller et al., 2006; Gilbert y Maalouf, 2008; Hudziak et al., 2004; Hudziak et al., 2006; Ivarsson y Larsson, 2008; Storch et al., (2006). Entre ellos destacan el de Geller et al. (2006) que demuestran una elevada sensibilidad (del 78\% al 92\%) y especificidad (del $86 \%$ al 94\%), así como un elevado poder diagnostico con un VPP de entre el $77 \%$ y el $86 \%$, y un VPN de entre el $90 \%$ y el $96 \%$, en función de los percentiles elegidos como punto de corte. También, destaca, el estudio de Ivarsson y Larsson (2008), en el que analizando la escala OCS-CBCL por medio de una curva ROC obtienen una $\mathrm{AUC}=0,91$.

Los resultados obtenidos por los diferentes estudios han favorecido la inclusión de la OCSCBCL como nueva escala en la última revisión de los autoinformes del CBCL, junto con la escala de estrés postraumático y la de tempo cognitivo lento (Achenbach y Rescorla, 2007; Achenbach et al., 2008).

El objetivo general de este estudio es valorar la utilidad de la OCS-CBCL para la detección del TOC en la infancia y la adolescencia en una muestra de nuestra población; de forma más específica se pretende evaluar el poder discriminativo de la escala determinando su consistencia interna, su sensibilidad, especificidad y valores predictivo positivo y negativo en la población que asiste a nuestro centro; y comparar los hallazgos con los resultados del estudio de validación (Nelson et al., 2001). 


\section{MÉTODO}

\section{Participantes}

Para este análisis participaron 78 padres de niños y adolescentes con edades comprendidas entre los 8 y los 17 años (Media $=13,2 ; D T=$ $2,6)$, de los cuales $54(69,23 \%)$ eran varones y $24(30,77 \%)$ eran mujeres; repartidos en tres muestras de igual tamaño $(n=26)$ y emparejados por edad y sexo.

Los grupos están formados por: a) grupo TOC, formado por padres de pacientes con TOC, clínicamente diagnosticados, mediante criterios DSM-IV-TR y CIE-10 MIA; b) grupo No-TOC, de padres de pacientes en tratamiento en el CSMIJ, por presentar trastorno psiquiátrico sin evidencia clínica de TOC (trastorno por déficit de atención con hiperactividad $24 \%$, trastornos depresivos $10 \%$, trastornos de ansiedad $24 \%$ y trastornos de adaptativos $42 \%$ ), y c) un grupo de padres control, de población general, seleccionados en sala de espera de pediatría (grupo PGral).

\section{Instrumento de evaluación}

Child Behavior Checklist (CBCL) (Achenbach, 1991). Cuestionario diseñado para abordar la definición de los problemas de conducta infantil de forma dimensional. Se basa en una cuidadosa revisión de la literatura y los datos recogidos de forma empírica a lo largo de diferentes años. Está diseñado para evaluar en un formato normalizado los problemas de comportamiento y las competencias sociales de los niños valoradas por los padres, tutores o los propios pacientes (en las versiones TRF, Teachers Rating Form, y YSR, Youth Self-Report, respectivamente).

El CBCL es un cuestionario auto o heteroaplicado, en el que los padres proporcionan información empírica sobre las competencias de sus hijos en 7 dimensiones conductuales a partir de las respuesta a 120 items relacionados con problemas de conducta que se puntúan en una escala de 3 puntos que van desde: «0: Falso, o Nunca», «1: De alguna manera o Algunas veces es verdad», «2: Muy cierto o Muy a menudo cierto». Consta también de 20 items que hacen referencia a competencia social para obtener información de los padres en relación a la cantidad y calidad de la participación de su hijo en deportes, pasatiempos, juegos, actividades, organizaciones, trabajos y tareas, la amistad, las relaciones sociales, y el rendimiento académico.

En el estudio actual se utilizan sólo las respuestas a cada uno de los items de la subescala OCS-CBCL (véase Tabla 1) y su puntuación total obtenida por medio del sumatorio de los 8 items, sin necesidad de corrección informatizada.

Tabla 1. Items de la OCS-CBCL de Nelson et al. (2001)

\begin{aligned} & \hline Ítem núm. \\ & \hline 9 «No puede sacarse de la mente ciertos pensamientos, obsesiones» \\ & 31 «Tiene miedo de que pueda pensar o hacer algo malo» \\ & 32 «Se siente como que tiene que ser perfecto/a» \\ & 52 «Se siente demasiado culpable» \\ & 66 «Repite ciertas acciones una y otra vez, compulsiones» \\ & 84 "Comportamiento raro» \\ & 85 «Tiene ideas raras» \\ & 112 «Está preocupado/a» \\ & \hline\end{aligned}

\section{Procedimiento}

En el protocolo de asistencia en las primeras visitas de nuestro CSMIJ, se entrega a todos los padres, a modo de cribado, el CBCL. Se revisó en la base de datos de nuestro servicio los pacientes que habían sido diagnosticados de TOC (según criterios de la Clasificación Internacional de Enfermedades CIE-10 MIA (WHO, 1996) y según criterios del Manual Estadístico y Diagnóstico de las Enfermedades Mentales DSM-IV-TR (APA, 2005) de entre los pacientes ambulatorios que habían recibido una primera visita en el periodo que comprende junio 
de 2005 y enero de 2010. Fueron identificados un total de 32 participantes de los cuales el $81,25 \%(N=26)$ tenían completado satisfactoriamente el cuestionario CBCL para padres.

El procedimiento de selección de la muestra se realizó a partir de 2 fuentes. La primera (grupos TOC y No-TOC) de población clínica ambulatoria asistida en el Centro de Salud Mental Infantil y Juvenil (CSMIJ) del distrito de Sant Martí del área metropolitana de Barcelona adscrito al sistema público de salud de la Generalitat de Catalunya. La segunda, (grupo PGral) de población general infanto-juvenil, seleccionados en sala de espera de pediatría de las áreas básicas de salud (ABS) de los centros de asistencia primaria (CAP) del sector de Ramón Turró y de Vila Olímpica.

Los grupos No-TOC y PGral fueron apareados por edad y sexo al grupo TOC.

\section{Análisis de datos}

Se ha realizado el análisis de los datos mediante el paquete estadístico PASW v.17.0. Mediante el coeficiente alfa de Cronbach se evalúa la consistencia interna de los 8 items de la subescala OCS-CBCL. Se obtienen las puntuaciones medias de la subescala OCS-CBCL en cada uno de los grupos y se comparan entre ellos mediante un análisis de la varianza (ANOVA), aplicando la prueba de comparaciones múltiples post-hoc de Scheffé. La validez de la subescala se calcula por medio de los índices diagnósticos básicos de Sensibilidad [verdaderos positivos / (verdaderos positivos falsos negativos)], la especificidad [verdaderos negativos / (verdaderos negativos falsos positivos)], PPV [verdaderos positivos / (verdaderos positivos falsos positivos)], y VPN [verdaderos negativos / (verdaderos negativos falsos negativos)].

Finalmente se establecen las curvas ROC con el fin de constatar la capacidad diagnóstica de la OCS-CBCL y se obtiene el AUC en tres grupos de análisis: a) TOC vs No-TOC y PGral, b) TOC vs no-TOC y c) TOC vs PGral.

\section{RESULTADOS}

\section{Estadísticos descriptivos}

La puntuación total de los 8 items de la OCS-CBCL presenta los siguientes descriptivos para cada uno de los 3 grupos: TOC, $M: 6,73$ (DT: 3,13); No-TOC, $M: 3,58$ (DT: 2,42); y PGral, $M: 2,54$ (DT: 0,91). El ANOVA pone de relieve la presencia de diferencias significativas entre los grupos $\left(F_{(2,75)}=22,56, p<0,001\right)$. Los contrastes posteriores de comparaciones múltiples entre medias (test pot hoc de Scheffé) muestran puntuaciones significativamente superiores en el grupo TOC en relación a los otros dos grupos (No-TOC y PGral), $p<0,001$; sin que las diferencias entre los grupos No-TOC y PGral sean significativas $(p=0,285)$.

Tabla 2. Matriz de intercorrelaciones entre los 8 items de la escala.

\begin{tabular}{|c|c|c|c|c|c|c|c|c|}
\hline Ítem núm. & 9 & 31 & 32 & 52 & 66 & 84 & 85 & 112 \\
\hline $\begin{array}{l}\text { 9. No puede sacarse de la mente cier- } \\
\text { tos pensamientos }\end{array}$ & - & & & & & & & \\
\hline $\begin{array}{l}\text { 31. Tiene miedo de que pueda pensar o } \\
\text { hacer algo malo }\end{array}$ & $0,30 * *$ & - & & & & & & \\
\hline $\begin{array}{l}\text { 32. Se siente como que tiene que ser } \\
\text { perfecto/a }\end{array}$ & 0,22 & 0,14 & - & & & & & \\
\hline 52. Se siente demasiado culpable & $0,31 * *$ & $0,31 * *$ & $0,25 *$ & - & & & & \\
\hline $\begin{array}{l}\text { 66. Repite ciertas acciones una y otra } \\
\text { vez, compulsiones }\end{array}$ & $0,33 * *$ & 0,05 & $-0,12$ & 0,18 & - & & & \\
\hline 84. Comportamiento raro & 0,03 & $0,35 * *$ & 0,05 & $-0,03$ & 0,14 & - & & \\
\hline 85. Tiene ideas raras & $0,28 *$ & $0,23 *$ & 0,08 & $0,25 *$ & 0,08 & $0,31 * *$ & - & \\
\hline 112. Está preocupado/a & $0,42 * * *$ & 0,16 & $0,29 *$ & $0,41 * * *$ & $0,36 * *$ & $-0,06$ & $-0,02$ & - \\
\hline
\end{tabular}

$* p<0,05 ; * * p<0,01 ; * * * p<0,001$. 


\section{Consistencia interna y correlación ítem-total}

Los resultados obtenidos muestran una consistencia interna (alfa de Cronbach $=0,65$ ) en los límites de lo aceptable, teniendo en cuenta el número de items de la escala y el tamaño de la muestra podrían estar subestimando el valor de alfa.

En la tabla 2 se presenta la matriz de correlaciones inter-items. Las correlaciones de los items con el total de la escala (ver Tabla 3 ) se sitúan por encima del valor 0,39 (ítem 84 ) hasta el valor 0,70 (ítem 9); estando todos los va- lores por encima del valor mínimo aceptable: 0,35 (Cohen y Manion, 1990).

Se ha calculado la sensibilidad, la especificidad, verdaderos positivos (VP), verdaderos negativos (VN), falsos positivos (FP), falsos negativos (FN), el valor predictivo positivo (VPP), el valor predictivo negativo (VPN) (véase Tabla 4) y las curvas ROC para la combinación de muestras: patología sin TOC y población general (Figura 1); patología sin TOC (Figura 2) y en comparación con el grupo de población general (Figura 3).

Tabla 3. Matriz de correlaciones ítem-total de la escala

\begin{tabular}{lc}
\hline Ítem núm. & Correlación ítem-total \\
\hline 9. No puede sacarse de la mente ciertos pensamientos & 0,70 \\
31. Tiene miedo de que pueda pensar o hacer algo malo & 0,55 \\
32. Se siente como que tiene que ser perfecto/a & 0,45 \\
52. Se siente demasiado culpable & 0,59 \\
66. Repite ciertas acciones una y otra vez, compulsiones & 0,52 \\
84. Comportamiento raro & 0,39 \\
85. Tiene ideas raras & 0,46 \\
112. Está preocupado/a & 0,63 \\
\hline
\end{tabular}

Nota: Todas las correlaciones son estadísticamente significativas $(p<0,001)$.

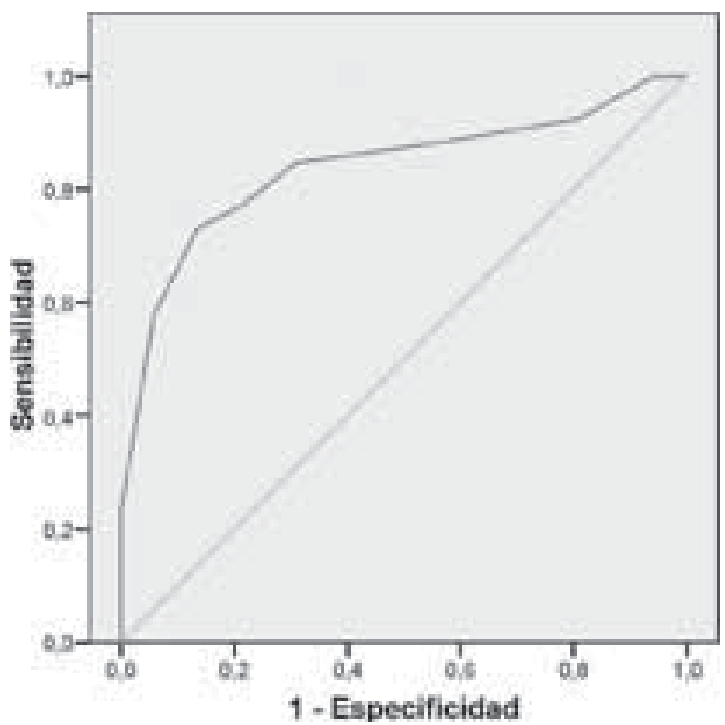

Figura 1. Curva ROC: TOC vs Muestra combinada (No-TOC + PGral) Nota: TOC: grupo de pacientes diagnosticados de trastorno obsesivo compulsivo. No-TOC: grupo de pacientes con otros trastornos psiquiátricos. PGral: grupo de población general

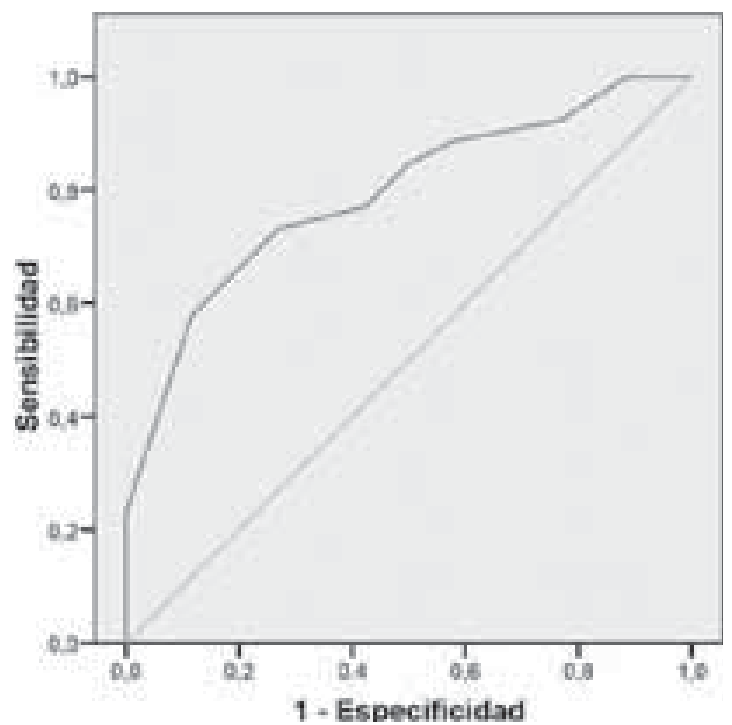

Figura 2. Curva ROC: TOC vs No-TOC

Nota: TOC: grupo de pacientes diagnosticados de trastorno obsesivo compulsivo. No-TOC: grupo de pacientes con otros trastornos psiquiátricos. 


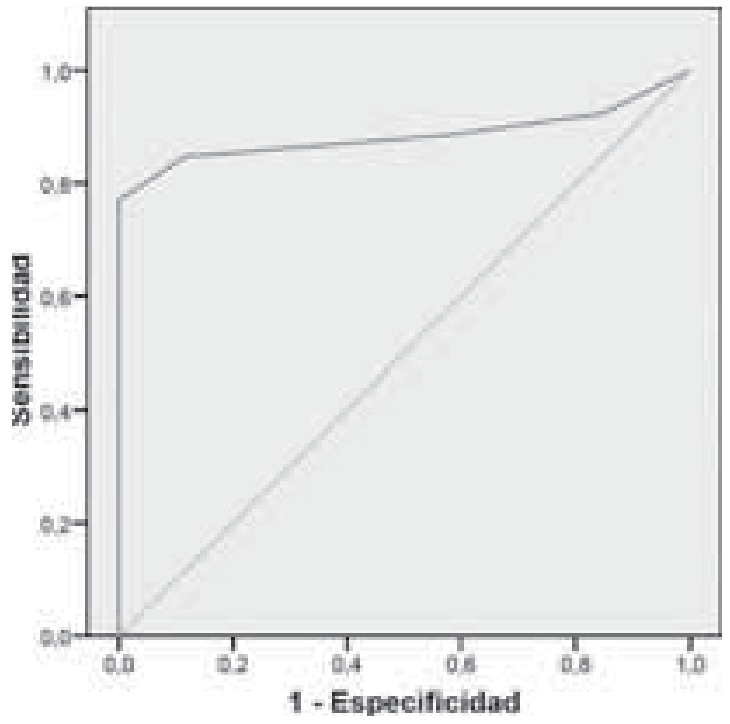

Figura 2. Curva ROC: TOC vs PGral

Nota: TOC: grupo de pacientes diagnosticados de trastorno obsesivo compulsivo. PGral: grupo de población general.
Utilizando los 2 puntos de corte más cercanos a la verdadera tasa de TOC en la muestra del estudio (percentiles 70 y 60 ), la sensibilidad es del $73,1 \%$ al $76,9 \%$, la especificidad del $78,8 \%$ al $86,5 \%$, VPP del $78,4 \%$ al $84,4 \%$ y VPN del 76,3\% al 77,3\%. La comparación directa con población general y asumiendo un punto de corte de 5, obtiene una sensibilidad de $76,9 \%$, una especificidad del $100 \%$, VPP de $100 \%$, y VPN de $77,3 \%$.

Se establece la puntuación directa de corte óptima que maximiza la ratio de VP y minimiza la ratio de FP, es decir, aquella en el que la distancia [(1-Sensibilidad) $2+$ (1-Especificidad) 2$]$ es menor (datos resaltados en Tabla 4). Cuando la muestra de pacientes con diagnóstico TOC se compara con población general el punto de corte óptimo es 5, al compararlo con las otras dos muestras (patológica no-TOC, y total: no-TOC+PGral) se requiere un punto de corte, más exigente, de 6.

Tabla 4. Tasas de VP, FP, VN, FN, VPP, VPN del grupo TOC en comparación con las otras muestras

\begin{tabular}{|c|c|c|c|c|c|c|c|c|}
\hline Percentil & $\begin{array}{c}\text { Punto } \\
\text { de Corte }\end{array}$ & $\begin{array}{c}\text { Sensibilidad } \\
\text { (VP) }\end{array}$ & $\begin{array}{c}1- \\
\text { Especificidad } \\
(\mathrm{FP})\end{array}$ & $\begin{array}{l}\text { Especificidad } \\
\text { (VN) }\end{array}$ & $\begin{array}{c}1- \\
\text { Sensibilidad } \\
(\mathrm{FN})\end{array}$ & VPP & VPN & Distancia \\
\hline \multicolumn{9}{|c|}{ Grupo TOC vs muestra combinada (No-TOC + PGral) } \\
\hline 50 & 3 & 88,50 & 57,70 & 42,30 & 11,50 & 60,53 & 78,62 & 0,35 \\
\hline 60 & 5 & 76,90 & 21,20 & 78,80 & 23,10 & 78,39 & 77,33 & 0,10 \\
\hline 70 & 6 & 73,10 & 13,50 & 86,50 & 26,90 & 84,41 & 76,28 & 0,09 \\
\hline 80 & 7 & 57,70 & 5,80 & 94,20 & 42,30 & 90,87 & 69,01 & 0,18 \\
\hline 90 & 8 & 34,60 & 1,90 & 98,10 & 65,40 & 94,79 & 60,00 & 0,43 \\
\hline 95 & 11 & 15,40 & 0,00 & 100,00 & 84,60 & 100,00 & 54,17 & 0,72 \\
\hline \multicolumn{9}{|c|}{ Grupo TOC vs Grupo No-TOC } \\
\hline 50 & 3 & 88,50 & 57,70 & 42,30 & 11,50 & 60,53 & 78,62 & 0,35 \\
\hline 60 & 5 & 76,90 & 42,30 & 57,70 & 23,10 & 64,51 & 71,41 & 0,23 \\
\hline 70 & 6 & 73,10 & 26,90 & 73,10 & 26,90 & 73,10 & 73,10 & 0,14 \\
\hline 80 & 7 & 57,70 & 11,50 & 88,50 & 42,30 & 83,38 & 67,66 & 0,19 \\
\hline 90 & 8 & 34,60 & 3,80 & 96,20 & 65,40 & 90,10 & 59,53 & 0,43 \\
\hline 95 & 11 & 15,40 & 0,00 & 100,00 & 11,50 & 100,00 & 54,17 & 0,72 \\
\hline \multicolumn{9}{|c|}{ Grupo TOC vs Grupo PGral } \\
\hline 50 & 3 & 88,50 & 57,70 & 42,30 & 11,50 & 60,53 & 78,62 & 0,35 \\
\hline 60 & 5 & 76,90 & 0,00 & 100,00 & 23,10 & 100,00 & 77,33 & 0,05 \\
\hline 70 & 6 & 73,10 & 0,00 & 100,00 & 26,90 & 100,00 & 76,28 & 0,07 \\
\hline 80 & 7 & 57,70 & 0,00 & 100,00 & 42,30 & 100,00 & 69,01 & 0,18 \\
\hline 90 & 8 & 34,60 & 0,00 & 100,00 & 65,40 & 100,00 & 60,00 & 0,43 \\
\hline 95 & 11 & 15,40 & 0,00 & 100,00 & 84,60 & 100,00 & 54,17 & 0,72 \\
\hline
\end{tabular}

Nota: TOC: pacientes diagnosticados de trastorno obsesivo compulsivo. No-TOC: pacientes con otros trastornos psiquiátricos. PGral: población general. En cursiva se señalan los puntos de corte óptimos. 
La precisión diagnóstica en referencia a cada grupo, junto a las estimaciones del AUC, el nivel de significación y los intervalos de confianza para un $I C=95 \%$ se pre- sentan en la Tabla 5. La mayor precisión se obtiene para el grupo de referencia de población general $(\mathrm{AUC}=0,88 ; I C=95 \%$ : 0,73 a $0,94)$.

Tabla 5. Áreas bajo la curva (AUC)

\begin{tabular}{|c|c|c|c|c|c|}
\hline \multirow[b]{2}{*}{$\begin{array}{l}\text { Variables resultado } \\
\text { de contraste }\end{array}$} & \multirow[b]{2}{*}{ Área } & \multirow[b]{2}{*}{$\begin{array}{l}\text { Error } \\
\text { típico }\end{array}$} & \multirow[b]{2}{*}{$\begin{array}{l}\text { Significación } \\
\text { asintótica }\end{array}$} & \multicolumn{2}{|c|}{$\begin{array}{l}\text { Intervalo de confianza } \\
\text { asintótico al } 95 \%\end{array}$} \\
\hline & & & & $\begin{array}{l}\text { Límite } \\
\text { superior }\end{array}$ & $\begin{array}{l}\text { Límite } \\
\text { inferior }\end{array}$ \\
\hline TOC vs No TOC y PGral & 0,838 & 0,055 & 0,000 & 0,730 & 0,945 \\
\hline TOC vs No-TOC & 0,791 & 0,063 & 0,000 & 0,668 & 0,914 \\
\hline TOC vs PGral & 0,884 & 0,056 & 0,000 & 0,775 & 0,993 \\
\hline
\end{tabular}

Nota: TOC: pacientes diagnosticados de trastorno obsesivo compulsivo. No-TOC: pacientes con otros trastornos psiquiátricos. PGral: población general

\section{DISCUSIÓN}

Los resultados obtenidos se hallan en la línea los del estudio de validación presentado por Nelson et al. (2001) y el de Geller et al. (2006) aportando datos a favor de su elevada sensibilidad y especificidad, de su favorable poder diagnóstico, en cuanto a elevados VPP y VPN.

Con el uso de las puntuaciones de corte correspondientes a los percentiles 60 y 70 , que son las que mejor reflejan la verdadera tasa de TOC en la muestra global (un tercio), la OCSCBCL muestra niveles satisfactorios de sensibilidad y especificidad y un adecuado poder diagnóstico tal y como indican los valores VPP y VPN en los pacientes con diagnóstico de TOC versus otros pacientes en tratamiento psiquiátrico ambulatorio sin TOC y ante participantes control, obtenidos de población general.

En general, los resultados obtenidos por la OCS-CBCL se comparan favorablemente con los del LOI-CV obtenidos por Rueda et al. (2007).

La subescala OCS-CBCL es útil en la medida en que el análisis de la curva ROC es muy bueno, ya que AUCs por encima de 0,75 indican que la capacidad diagnóstica de la prueba es apropiada. Aún así, nuestros resultados son sensiblemente inferiores a los obtenidos por Nelson (2001) y por Ivarsson y Larsson (2008). No obstante, cabe destacar que el nuestro es el primer estudio que se realiza en un centro no especializado en el diagnóstico del trastorno obsesivo-compulsivo; y en el que por tanto los padres tienen menor información que ayude a identificar de forma más franca los síntomas del trastorno en los items del CBCL.

En la misma línea que otros autores (Nelson et al., 2001) consideramos que la OCS-CBCL ofrece ciertas ventajas frente a otros cuestionarios (p.ej. el LOI-CV): 1) traducido a más de 85 lenguas, se ha utilizado en más de 7.000 publicaciones informando de su utilidad en 80 sociedades diferentes (Bérubé y Achenbach, 2008); 2) es uno de los instrumentos más utilizados en el ámbito clínico en la infancia y la adolescencia; 3) se ha demostrado ya su utilidad para la identificación de otros síndromes además del TOC, incluyendo enfermedades psiquiátricas comórbidas que se observan con frecuencia en el TOC (Achenbach, Dumenci y Rescorla, 2003; Bender, Auciello, Morrison, MacAllister y Zaroff, 2008, Ivanova et al., 2007a); 4) contiene un ítem que pregunta sobre «movimientos nerviosos o temblores» para identificar pacientes que están en riesgo de trastornos autoinmunes pediátricos neuropsiquiátricos asociados con estreptococos: síndrome PANDAS (pediatric autoimmune neuropsychiatric disorders associated with streptococci); 5) corrección mediante software informatizado a través del Achenbach System of Empirically Based Assessment, ASEBA); 6) sencillas instrucciones impresas que permite a los padres completar el cuestionario antes de 
la visita con el especialista, 7) dispone de cuestionarios homólogos (Achenbach y Rescola, 2001) para maestros, TRF (Ivanova et al., 2007b) y de modelo autoaplicado para jóvenes, YSR (Ivanova et al., 2007c) por medio de los cuales podrían obtenerse escalas paralelas (OCS-TRF y/o OCS-YSR).

Nuestros resultados deben interpretarse, no obstante, en el contexto de varias limitaciones metodológicas. En primer lugar, los diagnósticos de los diferentes pacientes se han recogido de forma retrospectiva y se realizaron de acuerdo con criterios clínicos (según criterios DSMIV y CIE 10) sin la utilización de entrevistas estructuradas o semiestructuradas que homogenicen los criterios entre los diferentes especialistas que realizaron los diagnósticos. En segundo lugar, y en comparación con los estudios anteriores, el tamaño de nuestra muestra es pequeño y no nos permite conclusiones más potentes. En tercer lugar, la muestra de nuestro grupo control de pacientes con trastorno psiquiátrico no-TOC, se ha elegido al azar, con el objetivo de limitar cualquier sesgo de evaluación, de entre el total de pacientes cuyos padres habían respondido el CBCL durante el proceso inicial de evaluación. Dado que el TOC está clasificado como un trastorno de internalización, se podría argumentar que una muestra compuesta por menos pacientes con trastornos de externalización y más con trastornos de ansiedad podrían haber proporcionado una mejor comparación; aspecto que no hemos tenido en cuenta.

Por último, aunque los resultados de las diferentes dimensiones del CBCL tienden a variar por edad y sexo, el tamaño de la muestra actual impide el examen de esta cuestión, en la escala OCS. Sería preciso poder valorar y determinar si se modifican los puntos de corte para diferentes grupos de edad, para lo que requeriríamos de una muestra más amplia.

A modo de conclusión, y dada la utilización generalizada del cuestionario CBCL para el screening de los trastornos mentales en la infancia y la adolescencia, los resultados de nuestro estudio apuntan la utilidad clínica y en investigación de la OCS-CBCL, para el cribado de la presencia de sintomatología obsesivacompulsiva en la infancia y la adolescencia.

\section{REFERENCIAS}

Achenbach, T.M. (1991). Manual for the Child Behaviour Checklist/4-18 and 1991 Profile. Burlington, VT: University of Vermont, Department of Psychiatry.

Achenbach, T.M., Becker, A., Döpfner, M., Heiervang, E., Roessner, V., Steinhausen, H.C., y Rothenberger, A. (2008). Multicultural assessment of child and adolescent psychopathology wiht ASEBA and SDQ instruments: research findings, applications, and futures directions. Journal of Child Psychology and Psychiatry, 49, 251-275.

Achenbach, T.M., y Dumenci, L. (2001). Advances in Empirically Based Assessment: Revised Cross-Informant Syndromes and New DSM-Oriented Scales for the CBCL, YSR, and TRF: Comment on Lengua, Sadowksi, Friedrich, y Fisher. Journal of Consulting and Clinical Psychology, 69, 699-702.

Achenbach, T.M., Dumenci, L., y Rescorla, L.A. (2003). DSM-oriented and empirically based approaches to constructing scales from the same item pools. Journal of Clinical and Adolescent Psychology, 32, 328-340.

Achenbach, T.M., y Rescorla L.A. (2001). Manual for the ASEBA School-Age Forms \& Profiles. Burlington, VT: University of Vermont, Research Center for Children, Youth, and Families.

Achenbach, T.M., y Rescorla, L.A. (2007). Multicultural Supplement to the Manual for the ASEBA School-Age Forms \& Profiles. Burlington, VT: University of Vermont, Research Center for Children, Youth \& Families.

Albores, L., Lara, C., Esperón, C., Cárdenas, J.A., Pérez, A.M., y Villanueva, G. (2007). Validez y fiabilidad del CBCL/6-18 (Incluye las escalas DSM). Actas Españolas de Psiquiatría, 35, 393-399.

Althoff, R.R., Rettew, D.C., Boomsma, D.I., y Hudziak, J.J. (2009). Latent class analysis of the Child Behavior Checklist Obsessive-Compulsive Scale. Comprehensive Psychiatry, 50, 584-592.

American Psychiatric Association (2005). Diagnostic and statistical manual of mental disorders (DSM-IV-TR). Washington D.C.: R.R. Donnelley \& Sons Company

Batlle, S., Raheb, C., Bielsa, A., Rafael, A., Tomàs, J., y Bassas, N. (2005). Evaluación psicológica del Trastorno Obsesivo Compulsivo en niños y adolescentes. En M. Casas y J. Tomas (Eds.), Trastorno obsesivo-compulsivo en la infancia y la adolescencia (pp. 133-159). Barcelona: Editorial Laertes.

Belloch, A., Cabedo, E., Morillo, C., Lucero, M., y Carrió, C. (2003). Diseño de un instrumento para evaluar las creencias disfuncionales del trastorno obsesivo-compulsivo: Resultados preliminares del Inventario de Creencias Obsesivas (ICO). International Journal of Clinical and Health Psychology, 3, 235-250.

Bender, H.A., Auciello, D., Morrison, C.E., MacAllister, W.S., y Zaroff, C.M. (2008). Comparing the convergent 
validity and clinical utility of the Behavior Assessment System for Children-Parent Rating Scales and Child Behavior Checklist. Epilepsy and Behavior, 13, 237-242.

Berg, C.Z., Whitaker, A., Davies, M., Flament, M.F., y Rapoport, J.L. (1988). The survery form of the Leyton Obsessionality Inventory child version: norms from an epidemiological study. Journal of the American Academy of Child and Adolescent Psychiatry, 27, 759-763.

Berg, C.Z., Whitaker, A., Davies, M., Flament, M.F., y Rapoport IM. (2002). Development of a short Leyton obsessional inventory for children and adolescents. Journal of the American Academy of Child and Adolescent Psychiatry, 41, 1246-1252.

Bernstein, G.A., Victor, A.M., Pipal, A.J., y Williams, K.A. (2010). Comparison of clinical characteristics of pediatric autoimmune neuropsychiatric disorders associated with streptococcal infections and childhood obsessive-compulsive disorder. Journal of Child and Adolescent Psychopharmacology, 20, 333-340.

Bérubé, R.L., y Achenbach, T.M. (2008). Bibliography of Published Studies Using ASEBA Instruments: 2008 edition. Burlington, VT: University of Vermont, Research Center for Children, Youth \& Families.

Black, D.W., y Gaffney, G.R. (2008). Subclinical obsessive-compulsive disorder in children and adolescents: additional results from a «high-risk» study. CNS Spectrums, 13, 54-61.

Bloch, M.H., Craiglow, B.G., Landeros-Weisenberger, A., Dombrowski, P.A., Panza, K.E., Peterson, B.S., y Leckman, J.F. (2009). Predictors of early adult outcomes in pediatric-onset obsessive-compulsive disorder. $\mathrm{Pe}$ diatrics, 124, 1085-1093.

Carter, A.S., Pauls, D.L., y Leckman, J.F. (1995). The development of obsessionality: continuities and discontinuities. En D.V. Cicchetti y D.J. Cohen (Eds.), Developmental psychopathology (pp. 609-632). New York: John Wiley and Sons.

Cohen, L., y Manion, L. (1990). Métodos de Investigación Educativa. Madrid: Editorial La Muralla.

Evans, D., Leckman, J., Carter, A., y Reznick, J. (1997). Ritual, habit, and perfectionism: The prevalence and development of compulsive-like behavior in normal young children. Child Development, 68, 58-68.

Flament, M., Whitaker, A., Rapoport, J.L., Davies, M., Berg, C.Z., y Schaffer, D. (1998). An epidemiological study of obsessive-compulsive disorder in children and adolescence. En J.L. Rapoport (Ed.), Obsessive-compulsive disorder in children and adolescents (1 ed., pp. 253-267). Washington: American Psychiatric Press.

García-Soriano, G., Belloch, A., y Morillo, C. (2008). Sobre la heterogeneidad del trastorno obsesivo-compulsivo: Una revisión. Revista de Psicopatología y Psicología Clínica, 13, 65-84.

Geller, D., Biederman, J., Faraone, S.V., Frazier, J., Coffey, B.J., Kim, G., y Bellordre CA. (2000). Clinical corre- lates of obsessive compulsive disorder in children and adolescents referred to specialized and non-specialized clinical settings. Depression and Anxiety, 11, 163-168.

Geller, D.A., Doyle, R., Shaw, D., Mullin, B., Coffey, B., Petty, C., Vivas, F., y Biederman, J. (2006). A quick and reliable screening measure for OCD in youth: reliability and validity of the obsessive compulsive scale of the Child Behavior Checklist. Comprehensive Psychiatry, 47, 234-240.

Gilbert, A.R., y Maalouf, F.T. (2008). Pediatric obsessivecompulsive disorder: management priorities in primary care. Current Opinion in Pediatrics, 20, 544-550.

Hanna, G.L. (1995). Demographic and clinical features of obsessive-compulsive disorder in children and adolescents. Journal of the American Academy of Child and Adolescent Psychiatry, 34, 19-27.

Helbing, M.L., y Ficca, M. (2009). Obsessive-compulsive disorder in school-age children. Journal of School Nursing, 25, 15-26.

Hudziak, J.J., Althoff, R.R., Stanger, C., Van Beijsterveldt, C.E., Nelson, E.C., Hanna, G.L., Boomsma, D.I., y Todd, R.D. (2006). The Obsessive Compulsive Scale of the Child Behavior Checklist predicts obsessivecompulsive disorder: a receiver operating characteristic curve analysis. Journal of Child Psychology and Psychiatry, 47, 160-166.

Hudziak, J.J., Van Beijsterveldt, C.E., Althoff, R.R., Stanger, C., Rettew, D.C., Nelson, E.C., Todd, R.D., Bartels, M., y Boomsma, D.I. (2004). Genetic and environmental contributions to the Child Behavior Checklist Obsessive-Compulsive Scale: a cross-cultural twin study. Archives of General Psychiatry, 61, 608-616.

Ivanova, M.Y., Achenbach. T.M., Rescorla, L.A., Dumenci, L., Almqvist, F., Bathiche, M., Bilenberg, N., Bird, H., Domuta, A., Erol, N., Fombonne, E., Fonseca, A.C., Frigerio, A., Kanbayashi, Y., Lambert, M.C., Leung, P., Xianchen, L., Minaei, A., Roussos, A., Simsek, Z., Weintraub, S., Wolanczyk, T., Zubrick, S., Zukauskiene, R., y Verhulst, F.C. (2007a). Testing the Teacher's Report Form syndromes in 20 societies. School Psychology Review, 36, 468-483.

Ivanova, M.Y., Achenbach. T.M., Rescorla, L.A., Dumenci, L., Almqvist, F., Bilenberg, N., Bird, H., Broberg, A.G., Dobrean, A., Döpfner, M., Erol, N., Forns, M., Hannesdottir, H., Kanbayashi, Y., Lambert, M.C., Leung, P., Minaei, A., Mulatu, M.S., Novik, T., Oh, K.J., Roussos, A., Sawyer, M., Simsek, Z., Steinhausen, H.C., Weintraub, S., Winkler M.C., Wolanczyk, T., Zilber, N., Zukauskiene, R., y Verhulst, F.C. (2007b). The generalizability of the Youth Self-Report syndrome structure in 23 societies. Journal of Consulting and Clinical Psychology, 75, 729-738.

Ivanova, M.Y., Dobrean, A., Dopfner, M., Erol, N., Fombonne, E., Fonseca, A.C., Frigerio, A., Grietens, H., Hannesdottir, H., Kanbayashi, Y., Lambert, M., Achenbach, T.M., Larsson, B., Leung, P., Liu, X., Minaei, A., Mulatu, M.S., Novik, T.S., Oh, K.J., Roussos, 
A., Sawyer, M., Simsek, Z., Dumenci, L., Steinhausen, H.C., Metzke, C,W., Wolanczyk, T., Yang, H.J., Zilber, N., Zukauskiene, R., Verhulst, F.C., Rescorla, L.A., Almqvist, F., Weintraub, S., Bilenberg, N., Bird, H., y Chen, W.J. (2007c). Testing the 8-syndrome structure of the CBCL in 30 societies. Journal of Clinical Child and Adolescent Psychology, 36, 405-417.

Ivarsson, T., y Larsson, B. (2008). The Obsessive-Compulsive Symptom (OCS) scale of the Child Behavior Checklist: a comparison between Swedish children with Obsessive-Compulsive Disorder from a specialized unit, regular outpatients and a school sample. Journal of Anxiety Disorders, 22, 1172-1179.

Ivarsson, T., Melin, K., y Wallin, L. (2008). Categorical and dimensional aspects of co-morbidity in obsessivecompulsive disorder (OCD). European Child and Adolescent Psychiatry, 17, 20-31.

King, R.A., y Noshpitz, J.D. (1991). Pathways of Growth: Essentials of Child Psychiatry. In J.D. Noshpitz (Ed.), Pathways of growth II (pp. 96-97). New York: Wiley \& Sons.

Leonard, H.L., Goldberger, E.L., Rapoport, J.L., Cheslow, D.L., y Swedo, S.E. (1990). Childhood rituals: Normal development or obsessive-compulsive symptoms? Journal of the American Academy of Child and Adolescent Psychiatry, 29, 17-23.

Lin, H., Williams, K.A., Katsovich, L., Findley, D.B., Grantz, H., Lombroso, P.J., King, R.A., Bessen, D.E., Johnson, D., Kaplan, E.L., Landeros-Weisenberger, A., Zhang, H., y Leckman, J.F. (2010). Streptococcal upper respiratory tract infections and psychosocial stress predict future tic and obsessive-compulsive symptom severity in children and adolescents with Tourette syndrome and obsessive-compulsive disorder. Biological Psychiatry, 67, 684-691.

Micali, N., Heyman, I., Perez, M., Hilton, K., Nakatani, E., Turner, C., y Mataix-Cols, D. (2010). Long-term outcomes of obsessive-compulsive disorder: follow-up of 142 children and adolescents. British Journal of Psychiatry, 197, 128-134.

Moriana, J.A., y Martínez, V.A. (2011). La psicología basada en la evidencia y el diseño y evaluación de tratamientos psicológicos eficaces. Revista de Psicopatología y Psicología Clínica, 16, 81-100.

Nelson, E.C., Hanna, G.L., Hudziak, J.J., Botteron, K.N., Heath, A.C., y Todd, R.D. (2001). Obsessive-compulsive scale of the child behavior checklist: specificity, sensitivity, and predictive power. Pediatrics, 108, E-14.

Rueda-Jaimes, G.E., Díaz-Martínez, L.A., Escobar-Sánchez, M., Franco-López, J.A., Navarro-Mancilla, A.A., y Cadena-Afanador, L.P. (2007). Validation of the short version of the Leyton obsessional inventory for children and adolescents in Bucaramanga, Colombia. Atención Primaria, 39, 75-80.

Sandín, B., Chorot, P., Valiente, R.M., y Lostao, L. (2009). Validación española del cuestionario de preocupación PSWQ: Estructura factorial y propiedades psicométricas. Revista de Psicopatología y Psicología Clínica, 14, 107-122.

Sardinero, E., Pedreira, J.L., y Muñiz, J. (1997). El cuestionario CBCL de Achenbach: adaptación española y aplicaciones clínico-epidemiológicas. Clínica y Salud, 8, 447-480.

Scahill, L., Riddle, M.A., McSwiggin-Hardin, M., Ort, S.I., King, R.A., Goodman, W.K., Cicchetti, D., y Leckman, J.F. (1997). Children's Yale-Brown ObsessiveCompulsive Scale: Reliability and validity. Journal of the American Academy of Child and Adolescent Psychiatry, 36, 844-852

Schrag, A., Gilbert, R., Giovannoni, G., Robertson, M.M., Metcalfe, C., y Ben-Shlomo, Y. (2009). Streptococcal infection, Tourette syndrome, and OCD: is there a connection? Neurology, 73, 1256-1263.

Storch, E.A., Larson, M.J., Muroff, J., Caporino, N., Geller, D., Reid, J.M., Morgan, J., Jordan, P., y Murphy, T.K. (2010). Predictors of functional impairment in pediatric obsessive-compulsive disorder. Journal of Anxiety Disorders, 24, 275-283.

Storch, E.A., Murphy, T.K., Adkins, J.W., Lewin, A.B., Geffken, G.R., Johns, N.B., Jann, K.E., y Goodman, W.K. (2006). The children's Yale-Brown obsessivecompulsive scale: psychometric properties of child-and parent-report formats. Journal of Anxiety Disorders, 20, 1055-1070.

Storch, E.A., Murphy, T.K., Bagner, D.M., Johns, N.B., Baumeister, A.L., Goodman, W.K., y Geffken, G.R. (2006). Reliability and validity of the Child Behavior Checklist Obsessive-Compulsive Scale. Journal of Anxiety Disorders, 20, 473-485.

Turner, C.M. (2006). Cognitive-behavioural theory and therapy for obsessive-compulsive disorder in children and adolescents: current status and future directions. Clinical Psychology Review, 26, 912-938.

Walitza, S., Melfsen, S., Jans, T., Zellmann, H., Wewetzer, C., y Warnke, A. (2011). Obsessive-compulsive disorder in children and adolescents. Deutsches Ärzteblatt International, 108, 173-179.

World Health Organization (1996). Multiaxial Classification of Child anb Adolescent Psychiatric Disorders. Cambridge, UK: Cambridge University Press. 